\title{
The benefits associated with respiratory co-located clinics
}

\author{
Authors: Martin Chadderton and Arvind Rajasekaran
}

\section{Aims}

The acute respiratory medicine directorate aimed to set up and sustain co-located clinics within primary care, with a focus on the multidisciplinary team model for long-term respiratory conditions.

\section{Methods}

An acute respiratory medicine consultant, in conjunction with a GP, ran a respiratory clinic for patients with long-term COPD and asthma within the GP's local practice.

A morning session was used where five patients were seen. A PDSA (plan do study act) cycle is to be used to advance this process this year; the results below are from PDSA cycle 1 .

\section{Results}

The table below illustrates the main benefits aggregated into a matrix. The cost improvements were calculated using reference costs, but actual pound $(\mathfrak{E})$ values have been removed.

Five patients were seen within the morning session. Five series of outpatient appointments, one emergency admission and one ultrasound were deemed to be avoided as a result. Medicine was rationalised and sharing of expertise were also benefits. An overall cost saving of circa $£ 3,000$ with a $£ 20$ recurring saving on drugs was made; the cost pressure of a morning's consultant and GP time was not deducted from this total, but would not have exceeded the other financial savings.

Table 1 shows the benefits of PDSA cycle 1 pilot. The patients' names have been changed.

\section{Conclusions}

The benefits from the initial PDSA cycle were predominately financial, in patient experience (patient feedback showed that they felt valued by having an acute specialist present) and in knowledge sharing (the combination of primary and secondary condition and treatment data had not previously been available). There was enough evidence that these benefits were replicable and improvable to continue with further PDSA cycles.

\section{Conflict of interest statement}

There are no known conflicts of interest.

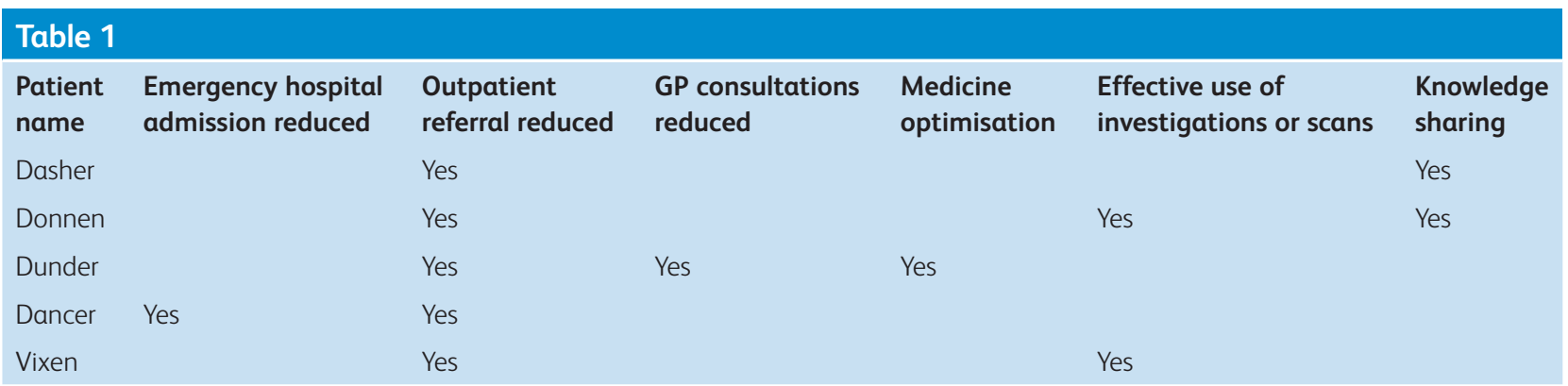

Authors: Sandwell and West Birmingham Hospitals NHS Trust,

West Bromwich, UK 